professionell Befaßten und von Gewalt Betroffenen deutlich geworden. Anscheinend entwickeln sich Gesetzgebung, Gerichte, Staatsanwaltschaft, Polizei, Beratungsstellen und Betroffene im Umgang mit Gewalt auseinander: Es bestehen unterschiedliche Interpretations- und Verhaltensmuster. Bedeutsam ist hier für die Zukunft ein Austausch aller Beteiligten, um einen Bewußtseinswandel gerade auch in der höchstrichterlichen Rechtsprechung zu erreichen. Ohne die Entwicklung eines vorurteilsfreien Umgangs mit Sexualdelikten können keine grundlegenden Veränderungen erzielt werden. Punktuelle Reformen würden lediglich zu neuen Umgehungsstrategien der Gerichte führen.

Der Gewaltbegriff muß im Hinblick auf das Rechtsgut der sexuellen Selbstbestimmung neu definiert werden. Bis heute wird von den Frauen "geschlechtsrollenkonformes Verhalten erwartet, z. B. bestimmte Situationen zu vermeiden. Diese vorrechtliche(n) Stereotypien schränken die Umsetzung des Strafrechts auf eine dem Opfer nicht dienliche Weise ein. Die Entkriminalisierung im Falle von Beziehungstaten macht private Gewalt zur Privatsache und führt zu fehlendem Schutz der sexuellen Selbstbestimmung. Ein öffentliches Interesse liegt jedoch vor und die Konsequenz sollte Strafverfolgung sein, da grundlegende individuelle Rechte verletzt werden.

\title{
Lorenz Petersen
}

\section{Zwischen Mythos und Misere: Die amerikanische Umweltbehörde EPA*}

\section{Einfübrung}

Umweltpolitik ist in die Jahre gekommen und mit ihr ihre staatliche Organisationsweise. Parallel zur institutionellen Etablierung des Umweltschutzes seit einer Aufbruchphase Anfang der siebziger Jahre haben sich Vorstellungen von der Beherrschbarkeit des Umweltproblems angesichts immer neuer krisenhafter Zuspitzungen und schleichender Gefährdungen stark relativiert. Ein prominentes Beispiel staatlicher Umweltschutzbemühungen ist die amerikanische Umweltbehörde EPA (Environmental Protection Agency), die seit ihrer Gründung 1970 eine ausgesprochen bewegte, wechselvolle Geschichte erlebte. Prominent heißt nicht auch gleich beliebt. Denn die Position der Behörde zwischen Kongreß und Weißem Haus, gerichtlicher Kontrolle und dem über Lobbygruppen kanalisierten Teil der öffentlichen Meinung ist ausgesprochen diffizil. Nicht selten resultierte aus dieser Konstellation eine phasenweise Lähmung der EPA-Aktivitäten in wichtigen umweltpolitischen Fragen.

Im internationalen Vergleich galten die »policies « US-amerikanischer Umweltpolitik vor allem in den siebziger Jahren als innovativ, und die EPA wurde und wird besonders von Ökonomen im Zusammenhang mit der Anwendung von Zertifikatslösungen in der Luftreinhaltepolitik gern zitiert. Um so mehr wurde die Haltung der Bush-Administration auf der UN-Umweltkonferenz in Rio de Janeiro zum Ausdruck für den geringen Stellenwert des Umweltschutzes gegenüber nationalen

* Gedankt sei an dieser Stelle dem Zentrum für Nordamerikaforschung in Frankfurt/Main und der Stiftung Volkswagenwerk fur die Gewahrung eınes insgesamt sechsmonatıgen Internship-Stipendiums, durch das der Autor im Winter/Fruhjahr 1990/91 Gelegenheit hatte, das "Innenleben « der Environmental Protection Agency kennenzulernen. 
ökonomischen Interessen, für die der damalige EPA-Chef William K. Reilly die Verteidigungslinie aufzubauen hatte. ${ }^{1}$

Im folgenden wird der Versuch einer Standortbestimmung unternommen, in der der Zustand der amerikanischen Umweltpolitik über ihre zentrale Institution auf Bundesebene, die EPA, erschlossen werden soll. Dazu soll zunächst ein Überblick gegeben werden zu Entstehung, Organisation und Aufgaben der Behörde. Es folgt eine Analyse der angedeuteten institutionellen "Gefangenschaft«, in der sich die EPA, mehr oder weniger stark, seit ihrer Gründung befindet. Am Beispiel des »emissions trading " - Programms widmet sich ein weiterer Abschnitt der Frage, welche Erfahrungen mit dem Einsatz ökonomischer Instrumente in der amerikanischen Umweltpolitik bislang gemacht wurden, bevor schließlich die Perspektiven für eine Neugewichtung und Umorientierung diskutiert werden.

\section{Entstehung}

Die Etablierung regulativ tätiger Bundesbehörden ging in den USA in zwei großen Etappen vor sich: Der erste Schub fand in den dreißiger Jahren im Zusammenhang mit der New-Deal-Politik Franklin D. Roosevelts statt, als mit der Federal Power Commission, der Food and Drug Administration, dem Federal Home Loan Board, der Federal Deposit Insurance Corporation, der Securities and Exchange Commission, der Federal Communications Comission, der Federal Maritime Commission und dem Civil Aeronautics Board die heute als »old-line agencies" geltenden Aufsichtsbehörden entstanden. ${ }^{2}$ Diese Gruppe machte zusammen mit Interstate Commerce Commission, mit Federal Reserve Board und Federal Trade Commission, die bereits vor dem New Deal bestanden, das »New Deal Regulatory Regime « aus, welches in erster Linie die Regulierung natürlicher Monopole’3 sowie die Bekämpfung unlauteren Wettbewerbs und unseriöser finanzieller Praktiken zum Ziel hatte und ohne wesentliche Veränderungen bis 1970 bestand. ${ }^{4}$

Diese regulative Landschaft veränderte sich einschneidend zum Ende der sechziger, Anfang der siebziger Jahre, als fast gleichzeitig Environmental Protection Agency, Occupational Safety and Health Administration, Consumer Product Safety Commission, Mining Safety and Health Administration und Nuclear Regulatory Commission begründet wurden. ${ }^{5}$ Anders als bei den existierenden »old-style«-Behörden ging es bei diesem zweiten Schub nicht um distributives Marktversagen oder Marktzugang, sondern um Gesundheit, Sicherheit, Umweltschutz - im weitesten Sinne »soziale« Problembereiche. ${ }^{6}$ Der Hauptunterschied zwischen diesen beiden Generationen von Bundesbehörden liegt darin, daß die jüngeren »social regulatory agencies« nicht mehr wie die »old-line agencies« ihre Arbeit weitestgehend im Stillen verrichten können, oft in enger Zusammenarbeit mit dem zu überwachenden Wirt-

I Vgl. Financial Times, Friday June s, 1992: "Summit's arch demon waves a sharp trident«, S.6.

2 Portney, Paul R. (1990): EPA and the evolution of federal regulation. In: Ders. (Hrsg.): Public policies for environmental protection. Washington D. C., S. 7

3 Als natürliche Monopole werden zumeist auf technischen Unteilbarkeiten beruhende Fälle zunehmender Skalenerträge wie zum Beispiel Telekommunikation, Wasser- und Energieversorgung, Eisenbahnen bezeichnet. Vgl. Streit, Manfred E. (199I): Theorie der Wirtschaftspolitik. Vierte Auflage. Düsseldorf, S. $88 \mathrm{f}$. "In such a case, it is argued, consumers will enjoy the lowest prices if one firm serves the whole market rather than sharing it with two or more competitors, as long as the single provider is regulated so as not to abuse its monopoly position. " Portney (Fn. 2), S. 23.

4 Portney (Fn. 2), S. 8; Hoberg, George (1992). Pluralism by design. Environmental policy and the American regulatory state. New York, Westport (Connecticut), London, S. 36.

5 Portney (Fn. 2), S. 7 .

6 White, Lawrence J. (1981): Reforming regulation: Processes and problems. Englewood Cliffs (New Jersey), S. 3 I ff. 
schaftsbereich und nur ab und zu auch mit dem zuständigen Ausschuß des Kongresses. ${ }^{7}$ Besonders die Occupational Safety and Health Administration (OSHA), zuständig für den Arbeitsschutz, und die EPA sind Beispiele für die Querschnittsorientierung und den weiten Kompetenzbereich der "neuen « Behördengeneration.

Institutionelle Neuerungen in der Form neuer Regierungsbehörden waren jedoch nur ein Ausdruck für weitreichendere gesellschaftspolitische Veränderungen, die nach Ansicht Hobergs zu einem "New Pluralist Regime« führten und mit einem »bemerkenswerten Umfang an Veränderungen in den Zielen, Aktoren, Prozessen und Institutionen regulativer Politik « verbunden waren. ${ }^{8}$ So kamen im Bereich Umweltpolitik zu den traditionsreichen Naturschutzverbänden Sierra Club (gegründet I892), Audubon Society (1905), Wilderness Society (1935) und National Wildlife Federation (1936) in einer Art Analogie zu den neuen staatlichen Institutionen Anfang der siebziger Jahre neue Umweltlobbygruppen wie der Environmental Defense Fund (EDF) und der Natural Resources Defense Council (NRDC) hinzu. ${ }^{9}$ Diese Gruppen mit einer sehr viel niedrigeren Mitgliederzahl als die traditionellen Naturschutzverbände finanzieren sich vor allem durch Spenden aus Stiftungsgeldern und machten von Beginn an konsequenten Gebrauch des 1970 vom Kongreß verabschiedeten »Citizen Suit«-Gesetzes, mit dem weitreichende Klagemöglichkeiten gegen Umweltverschmutzer selbst oder staatliche Behörden wegen unvollständiger Umsetzung von Umweltgesetzen eingeführt wurden. ${ }^{10}$ Die EPA nimmt bis heute immer wieder auf der gerichtlichen Anklagebank Platz, weil zeitliche Implementationsvorgaben in Gesetzen nicht eingehalten werden (können) oder weil sie aus dem Gesetzestext abgeleiteten »regulations « je nach zugrundeliegendem Interesse entweder als zu streng oder zu lax eingeschätzt werden. ${ }^{11}$

Ein zusätzlicher Grund für die Administration unter Präsident Nixon, im Bereich Umweltpolitik ein besonderes $\mathrm{Maß}$ an Aktivität zu entfalten, war das politische Gewicht Edmund Muskies, dem demokratischen Senator aus dem Bundesstaat Maine. Muskie hatte sich im Verlauf der sechziger Jahre als der Umweltpolitiker im Kongreß profiliert, und lange sah es so aus, als wäre er Nixons Gegenkandidat für die Wahlen um die Präsidentschaft $1972{ }^{12}$

Für die Gründung einer zentralen Umweltbehörde war kein nennenswerter politischer Widerstand zu überwinden. Die allseitige Unterstützung für die Behörde umfaßte eine breite Koalition einschließlich des Kongresses und führender Wirtschaftsvertreter, ${ }^{13}$ wobei die Gründe für diese Unterstützung sich vermutlich signifikant voneinander unterschieden. Während sich also die Frage nach der Existenz einer bundesstaatlichen Instanz für den Umweltschutz vergleichsweise leicht klären ließ, war die Gestalt dieser Institution durchaus nicht unumstritten.

Mit der Erarbeitung von Vorschlägen für die Konzipierung einer Bundesumweltschutzbehörde beauftragte Präsident Nixon 1969 eine Regierungskommission aus »professionellen Reformern $\aleph^{14}$ unter dem Vorsitz von Roy Ash. Der Vorsitzende des

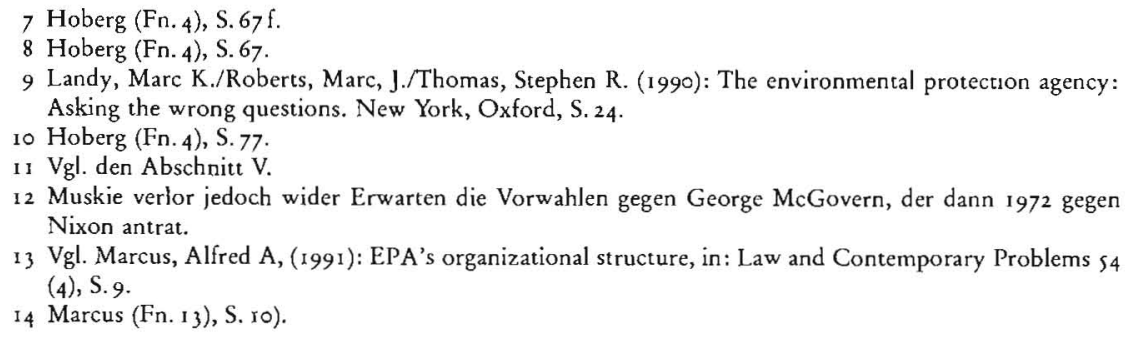


nach ihm benannten »Ash Council« verfolgte die Idee einer grundlegenden Reform des Regierungsapparates, in dem es nur noch vier Superministerien geben sollte: Community Development, Economic Affairs, Human Resources und Natural Ressources. ${ }^{15}$ Ein Department of Environment and Natural Resources entstand schließlich jedoch genausowenig wie die anderen drei Superministerien, vor allem wegen des Widerstandes aus den bestehenden Ministerien und weil es auch innerhalb des Ash-Councils erhebliche Zweifel an der von Ash selbst favorisierten Lösung gab. ${ }^{16}$ Die Senatoren Hugh Scott und Edmund Muskie brachten überdies einen Gesetzentwurf ein, der eine unabhängige, selbständige Umweltschutzverwaltung vorsah. ${ }^{17}$ Aus der Sicht Präsident Nixons hatte eine eigenständige Spezialbehörde ${ }^{18}$ zwei Vorteile: Sie war zugleich sichtbares Zeichen des Handelns in der Umweltpolitik und ein Kompromiß zwischen Befürwortern und Gegnern einer totalen Reorganisation der Regierung. Ironischerweise entstand so eine relativ machtvolle amtliche Institution für den Umweltschutz als ein Kompromiß zwischen Gruppen innerhalb der Administration, von denen keine ein ausgesprochener Verfechter von Umweltinteressen war. ${ }^{19}$

Am 9. Juli 1970 legte Nixon schließlich den »Reorganization Plan No. 3 « dem Kongreß zur Genehmigung vor und faßte damit Abteilungen aus den Departments of Interior, Health, Education and Welfare und Agriculture, aus Atomic Energy Commission, Federal Radiation Council und dem Council on Environmental Quality unter einem Dach zusammen. Im Dezember stimmte der Kongreß der Vorlage zu, und die neue Behörde nahm ihre Arbeit auf. ${ }^{20}$

\section{Organisation}

Weil die EPA bereits bestehende Programme und die für sie zuständigen Abteilungen aus den verschiedensten Teilen der Administration übernahm, war sie von Anfang an relativ groß. Die 5500 zunächst über ganz Washington verteilten Beamten konnten dann im März 1971 ein eigenes Gebäude im weniger feinen Südwesten Washingtons beziehen. ${ }^{21}$ Mit der Gründung der EPA war auch die Absicht verbunden, vorher administrativ oft nach Umweltmedien separierte Zuständigkeiten aufzulösen und unter einer integrierten, funktionalen Organisationsstruktur zusammenzufassen. Dazu sollten fünf Hauptabteilungen mit der Kompetenzaufteilung research, monitoring, standard setting, enforcement und assistence entstehen. Obwohl jedoch William Ruckelshaus, erster EPA-Chef ( Administrator «), durchaus von der grundsätzlichen Richtigkeit dieser integrierten Organisationsstruktur überzeugt war, setzte er sie nur teilweise auch in die Praxis um. ${ }^{22}$ Die Auflösung der bestehenden medialen oder auf bestimmte administrative Programme zugeschnittenen Abteilungen hätte möglicherweise erheblichen Verzug bei der Umsetzung der vom Kongreß in schneller Folge verabschiedeten Umweltgesetze bedeutet. Die erste formalisierte Organisationsstruktur der EPA enthielt so Hauptabteilungen, die nach

Is Landy/Roberts/Thomas (Fn. 9), S. 31.

16 Vgl. hierzu ausführlich Marcus (Fn. I 3), S. 10 ff. und Landy/Roberts/Thomas (Fn. 9), S. 30 ff.

17 Marcus, (Fn. I 3), S. Io.

$18 \mathrm{Im}$ Gegensatz zu anderen "regulatory agencies" wie etwa der OSHA, die in das Department of Labor eingegliedert wurde, 1st EPA direkt dem Prasidenten unterstellt.

19 Landy/Roberts/Thomas (Fn. 9), S. 32.

20 Portney (Fn. 2), S. 10.

21 Vgl. Lewis, Jack (1985): The birth of EPA, in: EPA Journal in (9), S. 6-1 I.

22 Davies, Terry (1990): The United States: Experıment and fragmentation, in: Haigh, N. lrwin, F. (Hrsg.): Integrated pollution control in Europe and North America. Washington D. C., S. $\$ 2$ und Marcus (Fn. I 3 ), S. $22 \mathrm{f}$. 
Umweltmedien (Luft und Wasser), nach Programmen (Pflanzenschutzregulierung, Strahlenschutz und Abfall) und funktional (Forschung und Kontrolle, Planung und Management sowie Umsetzung) abgegrenzt waren. ${ }^{23}$ Diese »hybrid structures « sind bis heute im wesentlichen erhalten geblieben trotz mehrerer Anläufe zur Umsetzung des funktionalen Organisationsprinzips. ${ }^{24}$

Die EPA besteht gegenwärtig aus elf Hauptabteilungen:

- International Activities

- Administration and Resource Management (interne Verwaltung)

- Enforcement (Überwachung und Durchsetzung)

- General Counsel (Rechtsabteilung)

- Policy, Planning, and Evaluation (strategische Abteilung)

- Inspector General (Revision)

- Water (Grund- und Oberflächengewässer)

- Solid Waste and Emergency Response (Abfall, Chemieunfälle, Superfund)

- Air and Radiation (Luft, Strahlenschutz)

- Pesticides and Toxic Substances (Pflanzenschutzmittelzulassung, Chemikalien)

- Research and Development (Forschung und Entwicklung).

Durch diese Form der Organisation mit einer Reihe von inhaltlichen Überlappungen zwischen den einzelnen Abteilungen sind die Zuständigkeiten oft nicht unmittelbar klar und erfordern ein großes Maß an behördeninterner Abstimmung. Um die Federführung für bestimmte Bereiche herrscht deshalb oft Konkurrenz. Für die meisten Problembereiche besitzen noch immer die an Umweltmedien orientierten Abteilungen einen deutlich größeren E.influß als die funktional abgegrenzten Abteilungen. Der Zuschnitt der entsprechenden Gesetze, die Struktur der Ausschüsse im Kongreß und die öffentliche Wahrnehmung verstärken ein mediales Problemverständnis. ${ }^{2 s}$

Trotz personeller und finanzieller Auszehrung zur Zeit der Reagan-Administration ${ }^{26}$ (198 r-88) beschäftigt die Behörde heute insgesamt etwa 17000 Menschen, von denen etwa ein Drittel in den über die gesamten USA verteilten zehn Regionalbüros oder diversen Forschungslabors arbeiten. ${ }^{26}$ Die EPA ist "Washington's largest regulatory agency in budget and personnel $\ll{ }^{27}$ Mit dem deutschen Umweltbundesamt ist die EPA wegen ihrer ausführenden Kompetenzen nicht zu vergleichen. Schon seit einigen Jahren wird in Regierung und Kongreß darüber diskutiert, die EPA in den Rang eines Ministeriums zu erheben.

23 Davies (Fn. 22), S. $22 \mathrm{f}$.

24 Marcus (Fn. 13), S. 23; und Landy/Roberts/Thomas (Fn. 9), S. 35.

25 Davies (Fn. 22), S. 53 .

26 Ronald Reagan hatte schon vor seiner Amtsubernahme 1981 auf Wahlkampfveranstaitungen seın Verstandnis von der Brisanz des Umweltproblems launig mit dem Laubfall im Herbst verglichen. Die mit jeder politischen Richtungsanderung im Weißen Haus verbundene Neubesetzung der EPA-Spitze machte Anne Gorsuch, konservative Anwaltin aus dem Bundesstaat Colorado, zur neuen Leiterin. Ihr Ziel war vor der Reform der Umweltpolitik eine Reform der Umweltbehörde. Bis zum März 1983, in ihrer gut zweijährigen Amtszeit, reduzierte sie die Zahl der Beschaftigten um ein Viertel, das damalige Jahresbudget sank von rund 700 auf 500 Millionen Dollar. Der Dauerzwist zwischen Leitung und Mitarbeitern war vorprogrammiert. Ihr vorzeitiger Rucktritt, vor allem wegen offentlich ausgetragener Strestigkeiten mit dem Kongreß, kam deshalb nicht uberraschend und wurde von den Mitarbeitern wie »die Befreiung von Paris" gefeiert. Die Folgen der personellen Radikalkur wirken noch heute nach: Ein großer Teil der Arbeit wird von "Contractors", vertraglich an EPA gebundenen privaten Beratungsfirmen ausgefuhrt. Mit dieser Zeitspanne der EPA-Geschichte beschaftigt sich ausfuhrlich Paul Portney (Hrsg.) (1984): Natural resources and the environment: The Reagan approach. Washington D.C.

26 Ettlin, Ross (1990): Facts to reflect on, in: EPA Journal 18 (6), S. 29.

27 Rosenbaum, Walter A. (1991): Environmental politics and policy. Second edition, Washıngton D.C., S. 97 . 
EPAs Aufgabe ist die Implementation umweltbezogener Gesetze. Das beinhaltet die Erarbeitung der Ausführungsbestimmungen (»regulations»), die Festsetzung von Standards sowie Kontrolle und Durchsetzung der rechtlichen Vorgaben ${ }^{28}$ Das Betätigungsfeld der Behörde ist bestimmt durch die gesetzgeberische Aktivität der letzten 20 Jahre, mit Clean Air Act, Clean Water Act, Noise Control Act, Safe Drinking Water Act, Resource Conservation and Recovery Act, Toxic Substances Control Act, Federal Insecticide, Fungicide, and Rodenticide Act und dem Superfund Act als den wichtigsten Gesetzen. ${ }^{29}$ Das zur Zeit bei rund fünf Milliarden U.S. \$ liegende Budget wird zum größten Teil für Gemeindezuschüsse zum Bau von Kläranlagen in den gesamten USA und die Sanierung von Altlasten unter dem Superfund-Programm aufgewendet. ${ }^{\circ}$ Die Aufsichts- und Kontrollaufgaben der EPA sind nicht auf den privaten Sektor beschränkt. Alle staatlichen, umweltrelevanten Aktivitäten fallen unter die Aufsicht der EPA, die dazu die nach dem National Environmental Policy Act von 1970 obligatorisch gewordenen Umweltverträglichkeitsstudien (Environmental Impact Statements) vorgelegt bekommt.

Eine besonders wichtige Rolle in der Umweltadministration der USA spieit das Verhältnis der Bundesstaaten zu den nationalen Regierungsinstitutionen. Die verfassungsrechtlich garantierte, durch die jeweils eigenständige Common-Law-Tradition unterstrichene Unabhängigkeit und gesetzgeberische Kompetenz der amerikanischen Bundesstaaten war während der siebziger Jahre durch eine weite Auslegung der in der Verfassung enthaltenen (»implied ") und daraus abgeleiteten (»resulting ") Aufgaben des Bundes zugunsten des Bundes in den Hintergrund getreten. ${ }^{31}$ Diese Tendenz hat sich in den vergangenen Jahren wieder umgekehrt, und eine eigene Gesetzgebung der Bundesstaaten wird auch bei vorliegender Bundeszuständigkeit akzeptiert, solange sie strenger und weitreichender ist als die Bundesgesetze. ${ }^{32}$ Die Bundesstaaten können sich ebenfalls von der EPA zur selbständigen Implementation der Bundesprogramme autorisieren lassen. Bedingung ist allerdings der Nachweis, $\mathrm{da} ß$ die nötigen gesetzlichen und administrativen Voraussetzungen erfüllt sind. Gleichwohl behält die EPA die Aufsicht über die Implementation auch in diesen Bundesstaaten. ${ }^{33}$ Vor allem die Zusammenarbeit mit den Institutionen auf bundesstaatlicher und lokaler Ebene ist die Aufgabe der zehn »Regional Offices«, die ganz ähnlich der Struktur der »headquarters« in Washington organisiert sind.

28 Vgl. Federal Publications Incorporated (Hrsg.) (1991): A practical guide to federal environmental Law. Reference manual. Washington D.C., S. I ff.

29 Vgl. Rosenbaum (Fn. 27), S. 97; zum Umweltrecht der USA: The Bureau of National Affarrs (Hrsg.) (1988): U.S. Environmental Laws. 1988 Edition. Washington D. C.; Wolf, Sidney M. (1988): Pollution Law Handbook: A guide to federal environmental laws. New York, Westport (Connecticut), London.

30 Rosenbaun (Fn. 27), S.97. Fur eine detaillierte Ubersicht der EPA-Aufgaben im einzelnen vgl. ebd., S. $98 \mathrm{f}$.

31 Hoberg (Fn. 4), S.87. Zum in der amerikanischen Verfassung festgelegten Verhaltnıs von Bund und Bundesstaaten vgl. Loewenstein, Karl (1959): Verfassungspraxis in den Vereinigten Staaten. Berlin, Gottingen und Heidelberg, S. 75 ff.; Rheinstein, Max (1976): United States of America, in: Knapp, V. (Hrsg.): International Encyclopedia of Comparative Law, Vol. i S-U i 38 und Mott, Richard N. (1990): Federalstate relations in U.S. environmental law: Implications for the European Community. EUI Working Papier EPU No. 9o/2. European University Institute, Florenz.

32 Marcus (Fn. 13), S. 33. Ein Beispiel sind die Emissionsstandards fúr Automobile in Kalifornien

33 Federal Publications Incorporated (Fn. 28), S. 2. 
Die EPA hat eine Schlüsselstellung in der amerikanischen Umweltpolitik. Das regulative Aufgabenfeld für die Behörde ist in der Tat enorm und wächst weiter. Nur insofern ist sie eine »machtvolle« Institution. Die ständig wachsende Aufgabenfülle bei einer gleichzeitig sich verschärfenden Kontrolle durch ein »pluralist regime« aus Kongreß, Weißem Haus und Gerichten ist ein Grund dafür, daß die EPA gemessen an ihren Vorgaben nur bescheidene Ergebnisse erzielt. ${ }^{34}$ Lazarus nennt diese Konstellation den »Cycle of Distrust «:35 Der Kongreß verabschiedet Umweltgesetze, deren Inhalt und Fristsetzungen oft außerordentliche Anforderungen an die Umsetzung stellen. Weil EPA die ihr gesetzten Ziele nicht erreicht, verabschiedet der Kongreß immer restriktivere Vorgaben, deren Umsetzung mit noch größerer Wahrscheinlichkeit nicht gelingt. Die theoretischen Erklärungsversuche für die Implementationsdefizite der EPA sind drei Varianten der »Agency Capture Theory «:

1. Die Neigung staatlicher Aufsichtsbehörden, sich mit ihrer Klientel zu verbünden.

2. Eine zu weitreichende Kompromißbereitschaft bei den Bediensteten der Behörde, die Umweltschutzziele »wegzuverhandeln «.

3. Die Gefangenschaft der Behörde in der eigenen Bürokratie selbst, die zuwenig Verständnis für die Interessen der von den Umweltschutzgesetzen wirtschaftlich Betroffenen aufbringt.

Ein Beispiel vorprogrammierten Scheiterns sind die Clean Air Act Amendments von 1970. Der Kongreß legte in ihnen fest, daß bis i97s nationale Luftqualitätsstandards (National Ambient Air Quality Standards NAAQS) erreicht sein sollten, die zugleich den Schutz der öffentlichen Gesundheit und wirtschaftlicher Interessen ("public welfare«) gewährleisteten. Der Kongreß schrieb außerdem vor, daß EPA innerhalb von 90 Tagen eine erste Liste »gefährlicher L Luftschadstoffe und in weiteren 180 Tagen für jeden Schadstoff einen Emissionsgrenzwert zum Schutz der öffentlichen Gesundheit vorzulegen habe. Der endgültige Grenzwert sollte dann in einer Frist von nochmals 180 Tagen abschließend bestimmt sein. ${ }^{36}$

Das Ausmaß dieser Aufgabe war immens. ${ }^{37}$ Wenig überraschend daher das Ergebnis: Weniger als is \% der Fristen konnten eingehalten werden, bis heute sind die Grenzwerte für die Luftqualität in vielen Regionen nicht umgesetzt, und die EPA ist nur für sieben von 274 bekannten Luftschadstoffen überhaupt aktiv geworden. ${ }^{37 a}$ Diskrepanzen dieser Größenordnung bei der Implementation von Gesetzen sind keine Einzelerscheinungen. Die EPA reagiert auf die von ihr oft als realitätsfern verstandenen und eher um die Wählerschaft als um die Problemlösung besorgte »symbolic legislation « des Kongresses mit Verzögerung oder dem Versuch, die Gesetzestexte pragmatisch anzulegen. ${ }^{38}$ Solche Strategien schaffen jedoch für die Behörde nur kurzfristige Entlastung immer weniger inhaltlichen Umsetzungsspielraum in der

34 Hoberg (Fn. 4), S. 208.

35 Lazarus, Richard J. (1991): The tragedy of distrust in the implementation of federal environmental law, in: Law and Contemporary Problems $54(4), S_{3} 314$ ff.

36 Lazarus (Fn. 35), S. 324 .

37 Lazarus beschreıbt die Situation wie folgt: "The administrative task was enormous. It required strict regulation of 20000 to 40000 major stationary sources of aur pollution, millions of cars and trucks being driven by average citizens, and 275 toxic air pollutants (sixty of which are known or suspected carcinogens), many of which were emitted by industries vital to local economies. In short, the act challenged not only susiness as usuak but slife as usual in the United States and demanded that EPA seek dramatic change in both", (Fn. 35), S. $3{ }_{4} 4 \mathrm{f}$.

37 Lazarus (Fn. 35), S. 325 .

38 Dwyer, John P. (1991): The pathology of symbolic legislation, in: Land Use and Environment Law Review 22, S. 296. 
Gesetzgebung. Andererseits konnten und können Umweltgruppen mit Prozessen gegen EPA aufgrund der offenkundigen Vollzugsdefizite mit Erfolg ersichtliche Verpflichtungen zur Umsetzung bestimmter Vorgaben erwirken. Die Rolle der umweltpolitischen Bremser übernahmen in diesem Cycle of Distrust zwei Organe: das Office of Management and Budget als der kontrollierende Arm des Weißen Hauses über die Tätigkeiten der eigenen Administration und das zu Zeiten der Bush-Präsidentschaft unter Umweltschützern berüchtigte Council on Competitiveness, eine kleine Gruppe aus Mitgliedern des Kabinetts unter dem Vorsitz von Vizepräsident Quayle, mit der Aufgabe, die wirtschaftlichen Konsequenzen der EPA-Politik zu evaluieren. Natürlich nutzen auch die ökonomisch begründeten Interessengruppen den erleichterten Zugang zu den Gerichten für die Bekämpfung der ihnen von EPA auferlegten Umweltvorschriften.

Die EPA wendet so einen großen Teil ihrer ohnehin begrenzten Ressourcen auf, um sich vor Gericht zu verteidigen, mit dem Office of Management and Budget und dem Weißen Haus zu verhandeln und ihre Entscheidungen vor Kongreßkomitees zu rechtfertigen. ${ }^{39}$ Das Selbstverständnis der EPA dagegen ist das einer »mission-oriented agency « die sich im Unterschied zu den »old-line agencies« nicht über den Bezug zu einem bestimmten Wirtschaftszweig definiert, sondern über eine gesellschaftspolitische Aufgabe. Die der Behörde gleichwohl unterstellte Gefangenschaft« in Partikularinteressen hilft so ironischerweise gerade das Bild herzustellen, das allenthalben beklagt wird: Bürokratie und Ineffizienz. Die EPA besitzt zwar weitreichende Kompetenzen in der Umweltpolitik, sie kann sie jedoch wegen einer Konstellation des »too much check, too little balance $\aleph^{40}$, durch die enge Kontrolle von Kongreß, Weißem Haus und Gerichtsbarkeit nicht wirksam einsetzen.

Seitdem die EPA 1970 ihre Arbeit aufnahm, sind die mit ihrer regulativen Tätigkeit verbundenen volkswirtschaftlichen Kosten staatlicher Umweltpolitik Gegenstand einer sich im Laufe der Zeit verschärfenden Kontroverse. Gefährden die EPA-Vorschriften Arbeitsplätze und die Existenzfähigkeit von Produktionsstandorten durch ineffiziente, irrationale und widersprüchliche Vorgaben?"1 Eine Reaktion auf das Kostenargument war die verstärkte Kontrolle der Regulierungsaktivitäten der eigenen Administration durch das Office of Management and Budget noch unter den Präsidenten Ford (1973-76) und Carter (1977-80), vor allem aber in der Amtszeit Reagans. ${ }^{42} \mathrm{Zu}$ den ersten Amtshandlungen des gerade in sein Amt eingeführten Präsidenten Reagan gehörte dementsprechend im Februar $198 \mathrm{r}$ ein Erlaß, nach dem für alle neuen Umweltvorschriften mit vermuteten volkswirtschaftlichen Kosten von mehr als I00 Mio US \$ obligatorische »Regulatory Impact Analyses« (RIA's) durchzuführen waren. Staatliche Vorgaben, deren potentieller Nutzen nicht die von ihnen verursachten Kosten überstieg, sollten unterbleiben. ${ }^{43}$ Die Verpflichtung, eine Kosten-Nutzen-Analyse zum Kriterium umweltpolitischer Vorgaben zu machen, stellte und stellt die EPA vor erhebliche Probleme, vor allem durch die oft unzureichenden Informationsgrundlagen zur Schätzung des volkswirtschaftlichen Nutzens. Daß es hier erhebliche Einschätzungsunterschiede gibt, belegen die chronisch weiten Differenzen zwischen den Schätzungen aus verschiedenen Teilen der Administration oder zwischen der EPA und der betroffenen Industrie. ${ }^{44}$

\footnotetext{
39 Lazarus (Fn. 35), S. 313.

$40 \mathrm{Vgl}$. Rosenbaum (Fn. 27), S. I 12.

4I Rosenbaum (Fn. 27), S. I 28.

42 Hoberg (Fn. 4), S. 200.

43 Rosenbaum (Fn. 27), S. 132.

44 Vgl. Rosenbaum (Fn. 27), S. $129 \mathrm{ff}$.
} 
Die unzureichende Berücksichtigung der mit ihr verbundenen Kosten ist eines der Hauptargumente von umweltökonomischer Seite gegen bisher praktizierte Formen regulativer Umweltpolitik. Statt mit außerordentlichem Aufwand das ohnehin nicht erreichbare Ziel einer risikolosen, verschmutzungsfreien Umwelt zu verfolgen, sollen sozialer Nutzen und soziale Kosten miteinander in Einklang gebracht und für jedes angestrebte Ziel die kostengünstigste Lösung gesucht werden. ${ }^{45}$ Die Mittel dazu sind das Eigeninteresse mobilisierende Anreizstrukturen statt starren "command and control«-Vorschriften. Die von Umweltökonomen schon früh ins Gespräch gebrachten Mittel der Wahl zur Steigerung der Effizienz staatlicher Umweltpolitik sind in erster Linie Abgaben- und Zertifikatslösungen.

Das Kostenargument und die regulative Überlast veranlaßten die Behörde schon früh, instrumentelle Alternativen zum »command and control « zu diskutieren und anzuwenden. Die EPA hat seit mehr als einem Jahrzehnt Erfahrungen mit Zertifikatsmodellen in der Luftreinhaltung gesammelt, an deren Einsatz in erster Linie von wissenschaftlicher Seite große Erwartungen geknüpft wurden. ${ }^{46} \mathrm{Am}$ Beispiel »emissions trading " zur Umsetzung des Clean Air Act sollen deshalb nach der Beschäftigung mit der »institutionellen Aufhängung* der Behörde auch instrumentelle Innovationsversuche beleuchtet werden. ${ }^{47}$

\section{Emissions trading}

Emissions trading bedeutet den Handel von Emissionsrechten in der Luftreinhaltepolitik, sowohl zwischen Unternehmen wie auch innerhalb eines einzelnen Betriebes. Gehandelt werden »emission reduction credits « (ERC's), die dadurch entstehen, daß Unternehmen in ihren Emissionen unterhalb eines ihnen zugewiesenen »baseline level « bleiben, einer Art Verschmutzungskontingent, das ihnen von der jeweiligen Umweltbehörde des betreffenden Bundesstaates zugewiesen wird.

Nach dem Clean Air Act bestimmt die EPA die Standards für die Luftqualität und legt sowohl Emissions- wie auch Immissionsgrenzwerte fest, deren Umsetzung jedoch in Händen der Bundesstaaten liegt. ${ }^{4^{8}}$ Die ausführenden Bundesstaaten legen ihre "state implementation plans (SIP's), der EPA zur Genehmigung vor. In den SIP's werden Anforderungen für die Genehmigungspraxis festgelegt. Für »major sources « sind generell Genehmigungsverfahren vorgesehen, während »minor sources« weniger strengen, von Bundesstaat zu Bundesstaat unterschiedlichen Anforderungen unterliegen. ${ }^{49}$ Die einzuhaltenden Auflagen sind in der Regel für Neuanlagen strenger als für bereits bestehende Anlagen und hängen außerdem davon $a b$, ob es

45 White, Lawrence J. (1981): Reforming regulation. Processes and problems, Englewood Cliffs (New Jersey), S. $35 \mathrm{ff}$.

46 "Until recently, market incentives have been more the province of academic economists than legislators and policy makers." Alm, Alvin L. (1992): A need for new approaches, in: EPA Journal 18 (2), S. 9.

47 Dabeı werde ich mich in erster Lınıe auf eine Studie von Robert W. Hahn und Gordon L. Hester von 1989 beziehen: Where did all the markets go? An analysis of EPA's emıssion trading program, in: Yale Journal on Regulation 6(x), S. I09-1 53, Handelbare Umweltzertifikate werden in den USA außerdem eingesetzt für Blei in Automobilkraftstoffen, fur Fluorchlorkohlenwasserstoffe und seıt kurzem auch für Schwefeldioxid. Stavins, Robert N. (1992): Harnessing the marketplace. We have to do more with less, in: EPA Journal I 8 (2), S. 28); einen ausgezeıchneten Überblick zur Diskussion uber den Einsatz umweltokonomischer Instrumente in den USA bietet Stavins, Robert N. (Hrsg.) (1991): Project 88 - round II. Incentives for action: Designing market-based environmental strategies. Sponsored by Senators Tim E. Wrrth and John Heinz. Washington D. C.

$4^{8}$ Für sieben Luftschadstoffe kann emissions tradıng angewendet werden: Staub, Schwefeldıoxid, Kohlenmonoxid, Stickoxide, Ozon, Blei und Kohlenwasserstoffe. Hahn/Hester (Fn. 47), S. I 14.

$49 \mathrm{Hahn} /$ Hester (Fn. 47), S. I Is. 
sich um eine Region handelt, deren Luftbelastung über den Immissionsgrenzwerten liegt (nonattainment area)..$^{\circ}$ Vier Modelle des emissions trading in der Luftreinhaltepolitik finden seit längerem Anwendung: ${ }^{51}$

- offsets

Eine neue major source« soll in einer nonattainment area angesiedelt werden. Die neu hinzukommenden Emissionen können durch Reduzierungen in bereits bestehenden Anlagen in mindestens gleicher Höhe ausgeglichen werden. Die dazu nötigen emission reduction credits können aus dem eigenen Unternehmen oder durch Zukauf von anderen Unternehmen aus der gleichen Luftreinhalteregion stammen.

\section{- bubbles}

Eine imaginäre Glocke über mehrere existierende Emissionsquellen ist die zugrundeliegende Vorstellung beim bubble-Modell. Unterhalb dieser Glocke können Unternehmen selbst entscheiden, wo sie den Schadstoffausstoß reduzieren, das heißt, es interessiert lediglich die Gesamtmenge eines emittierten Schadstoffes und nicht die Verteilung auf einzelne Quellen. Bubbles können für mehrere Quellen eines Unternehmens, aber auch mehrerer Unternehmen konstruiert werden, was durch den Handel der Emissionsrechte dazu führen soll, daß der Schadstoffausstoß dort reduziert wird, wo dies am kostengünstigsten möglich ist.

\section{- banking}

Unter dem banking-Modell können Unternehmen Emissionsrechte »sparen«. Das bedeutet, Guthaben an Verschmutzungsrechten sind auch über einen längeren Zeitraum gültig, können potentiell sogar im Wert steigen.

\section{- netting}

Für den Fall, daß ein Unternehmen den Schafstoffausstoß bei einer bestimmten Quelle innerhalb des Betriebes steigern will, besteht die Möglichkeit, die Klassifikation dieser Schadstoffquelle als "major source« zu verhindern, indem an anderer Stelle innerhalb des Betriebs soviel an Schadstoffen der gleichen Art eingespart wird, daß »netto " trotz Steigerung des Schadstoffausstoßes der Grenzwert zur Klassifikation als "major source" nicht überschritten wird.

Der Effekt der emissions-trading-Programme war bescheidener als erwartet. Nur eine begrenzte Zahl von Unternehmen hat von den mit dem Programm verbundenen Möglichkeiten Gebrauch gemacht. Die Auswirkungen auf die Umweltqualität sind daher auch kaum nennenswert. ${ }^{52}$ Allerdings haben sich für die am emissions trading teilnehmenden Unternehmen durchaus beachtliche Kosteneinsparungen ergeben von geschätzten mehreren Milliarden US $\$ .{ }^{33}$ Als am beliebtesten erwiesen sich das netting und die offsets mit jeweils mehreren tausend Anwendungen über die gesamten USA. ${ }^{54}$ Das ist im Falle der offsets deshalb nicht verwunderlich, weil offsets für den Bau neuer Schadstoffquellen in nonattainment areas zwingend vorgeschrieben sind. Eine Erklärung für die Beliebtheit des netting ist die Vermeidung von Verzöge-

so Hahn/Hester (Fn. 47), S. 114 f.

(I Morgenstern, Richard D. (1992): The market based approach at EPA, in EPA Journal 18 (2), S. 27 f.; vgl.

fur eine ausfuhrliche Auseinandersetzung auch bei Liroff, Richard A. (1986): Reforming air pollution regulations: The toil and trouble of EPA's bubble. Washington D.C.

\$2 Zum Umweltqualitatsaspekt nimmt insbesondere Liroff (Fn. si) Stellung

$53 \mathrm{Hahn} /$ Hester (Fn. 47), S. $136 \mathrm{ff}$.

54 Morgenstern (Fn. SI), S. 28. 
rungen durch den aufwendigen Genehmigungsprozeß für eine »major source«.5s Die Zahlen der Anwendung des bubble-Modells sind da sehr viel bescheidener: Bis I 986 sind 42 "Blasen « von der EPA selbst genehmigt worden und weitere 89 durch die Bundesstaaten unter von der EPA genehmigten Anwendungsvorschriften. ${ }^{56}$ In dem seither vergangenen Zeitraum sind nur sehr wenige bubbles eingerichtet worden, was sich möglicherweise durch die Novellierung des Clean Air Act von 1990 und seine Umsetzung bald ändern könnte. ${ }^{77}$ Noch geringer war die banking-Aktivität. Als Maßnahme zur Erleichterung eines tatsächlichen Handels mit Emissionsrechten konzipiert, zeigte sich, daß gerade dieses Ziel nicht erreicht wurde: Weniger als 20 Geschäfte mit Emissionsrechten zwischen Unternehmen schätzen Hahn und Hester und weniger als 100 unternehmensinterne banking-Anwendungen..$^{8}$

Hahn und Hester stellten insgesamt fest, daß der ganz überwiegende Teil des emissions trading auf unternehmensinternem Schadstoffmengenausgleich beschränkt bleibt: Eine aufwendige Suche nach Geschäftspartnern und der Genehmigungsvorbehalt der jeweiligen Umweltbehörden haben insbesondere bei dem zu großen Teilen unter EPA-Aufsicht stehenden bubble-Modell möglicherweise abschreckend gewirkt. ${ }^{59}$ Die ökonomische Erklärung dazu lautet, daß die Transaktionskosten für die Etablierung tatsächlicher Märkte an Emissionsrechten von den Unternehmen als zu hoch eingeschätzt wurden. ${ }^{60}$ Die relative Attraktivität der netting- und offsetProgramme ist auch durch die Altanlagenprivilegierung begründet, die so teilweise aufgehoben wird. ${ }^{61}$ Wahrscheinlich wichtigster Grund für das Fehlen eigentlicher Märkte an Emissionsrechten ist die Unsicherheit über die mit den ECR's tatsächlich verbundenen Eigentums- und Verfügungsrechten. Wie wird sich die regulative Situation im Zeitablauf entwickeln, was werden die Verschmutzungsrechte tatsächlich wert sein? Diese Zweifel sind plausibel, denn gerade das Einräumen von Verfügungsrechten (property rights) an Verschmutzung ist in der umweltpolitischen Diskussion sehr umstritten. ${ }^{62}$

Tatsächlich bringt die Vergabe der Verschmutzungsrechte, die in den oben beschriebenen Modellen als »baseline level« Berechnungsgrundlage für die ERC's ist, aber auch ein praktisches Problem mit sich: Die von den bundesstaatlichen Behörden per "grandfathering " ${ }^{63}$ also kostenlos anhand von Emissionswerten aus der Vergangenheit an die einzelnen Betriebe vergebenen baselines, liegen aufgrund mangelhafter Informationsgrundlage oft erheblich über den tatsächlichen Emissionswerten der Betriebe. Dadurch werden die emission reduction credits, die eigentlich aus einer Reduzierung der Schadstoffbelastung resultieren sollten, als "paper credits « unfreiwillig verschenkt. ${ }^{64}$ Tietenberg sieht demgemäß die "gemeinsame Wurzel der Probleme bei der Umsetzung der emissions-trading-Programme im Fehlen eines entsprechenden Überwachungssystems der Luftqualität. ${ }^{65}$

\footnotetext{
is Hahn/Hester (Fn. 47), S. 137 f.

56 Hahn/Hester (Fn. 47), S. 128 ; Morgenstern (Fn. 5I), S. 28

57 Morgenstern (Fn. 5 I), S. 28

$58 \mathrm{Hahn} /$ Hester (Anm. 47), S. 138

59 Hahn/Hester (Anm. 47), S. 137

60 Hahn/Hester (Anm. 47), S. 140.

61 Hahn/Hester (Anm. 47), S. 139.

62 Steven Kelman bietet eine interessante, psychologisch begrundete Kritik umweltokonomischer Instrumente: Kelman, Steven (198I): What price incentives? Economists and che environment. Boston.

$63 \mathrm{Vgl}$. Tietenberg, Thomas H. (1985): Emissions trading, an exercise in reforming pollution policy. Washington D.C., S. I 21 .

64 Hahn/Hester (Fn. 47), S. 1 I 4 ff.

65 Tietenberg (Fn. 63), S. 119
} 
Interessanterweise machte unter anderem die kostenlose Vergabe und zumindest theoretische Aussicht auf Profite das Zertifikatsmodell anders als die von den meisten amerikanischen Ökonomen sehr viel stärker favorisierten Abgabenlösungen in den USA politisch überhaupt erst durchsetzbar. ${ }^{66}$ Weitere politische Vorteile des emissions trading gegenüber Abgabenlösungen war die Möglichkeit einer graduellen Einführung, das Festhalten am System der Grenzwerte und nicht zuletzt die Tatsache, daß ein Zertifikatsmodell in den Umweltausschüssen des Kongresses verhandelt wird, jede Form von Abgabe jedoch mit wenig Aussicht auf Erfolg den Finanzausschuß des Senates und das "Ways and Means Committee« des Repräsentantenhauses zu passieren hätte. ${ }^{67}$

\section{Risiko als Grundlage der Umweltpolitik}

Auch wenn die tatsächliche Bedeutung umweltökonomischer "policies« in den Vereinigten Staaten bei weitem nicht so groß ist, wie es der Umfang an wissenschaftlicher Literatur zu diesem Thema suggeriert: Innerhalb der EPA hat Effizienz als Kriterium in der Umweltpolitik an Bedeutung gewonnen. Insbesondere die für Strategieentwicklung und Evaluierung zuständige Abteilung des Assistant Administrator for Policy, Planning and Evaluation ist auch personell fest in der Hand von Ökonomen. ${ }^{68}$ Unter anderem dadurch hat sich das Verständnis von der eigenen "Mission« im Laufe der Zeit gewandelt: Die Anfänge der Behörde unter dem ersten Administrator Ruckelshaus zu Beginn der siebziger Jahre waren vom optimistischen Glauben an die technischen Voraussetzungen zur Beherrschung der Umweltprobleme mit einer »end of pipe «-Umweltpolitik bestimmt. ${ }^{69}$ Unter Douglas Costle, dem Amtschef zur Zeit der Carter-Präsidentschaft, stand öffentliche Gesundheitsvorsorge an erster Stelle der Prioritätenliste in der Behörde mit dem relativen Krebsrisiko als allgegenwärtigem Maßstab zur Bewertung von Umweltproblemen. ${ }^{70}$ Die Behördenstrategie des EPA-Chefs unter Präsident Bush, Reilly, versuchte sich von diesen Vorstellungen zu lösen und eine umfassender ausgerichtete, auf wissenschaftlicher Basis entwickelte Prioritätensetzung in der Umweltpolitik anzusteuern. Dazu beauftragte Reilly ein wissenschaftliches Beratergremium, das Science Advisory Board, dessen Bericht vom September 1990 folgende Kernaussagen enthält: $7^{71}$ I. EPA sollte seine Anstrengungen da konzentrieren, wo die Möglichkeiten einer Minderung der Umweltrisiken am größten sind. Umweltprobleme wie Vernichtung von Lebensräumen, Ausrottung von Arten, Zerstörung der Ozonschicht und der Treibhauseffekt werden hier als »relatively high-risk problems eingestuft. Umweltprobleme mittlerer Risikostufe sind Pflanzenschutzmitteleinsatz, Verschmutzung von Oberflächengewässern, Saurer Regen und Luftschadstoffe, während zum Beispiel Tankerunglücke und Grundwasserverschmutzung als »relati-

66 Nelson, Robert H. (1987): The economics profession and the making of public policy, in: Journal of Economic Literature 25 (I), S. $68 \mathrm{ff}$.

67 Nelson (Fn. 66), S.68 f. Vgl, dazu auch die Ergebnisse von Kelmans Befragung uber den Einsatz okonomischer Instrumente in der Umweltpolitik bei Mitarbeitern von Kongreßausschussen, Umweltschuitzern und Vertretern der Industrie. Vgl. Kelman (Fn. 62), S. 93-124.

68 In den USA gilt "policy analysis s seit den siebziger Jahren sowohl an den Universitaten wie auch als Berufsbezeichnung ganz generell als Domane angewandter mikrookonomischer Theorie. Vgl. Kelman (Fn. 62), S. 5. Vgl. zur Bedeutung der ökonomischen Perspektive innerhalb von EPA Fraas, Arthur (1991): The role of economic analysis in shaping environmental policy, in: Law and Contemporary Problems 54 (1), S. I13-125.

69 Lewis (Fn. 21), S. $6 \mathrm{ff}$.

70 Landy/Roberts/Thomas (Fn. 9) S. 4 I.

7I Environmental Protection Agency (Hrsg.) (1990): Reducing risk: Setting priorities and strategies for environmental protection. Report of The Science Advisory Committee, Washıngton D. C. 
vely low risk problem « gelten. Vor allem aber sollten die Umweltrisiken nicht mehr wie in der Vergangenheit nach dem Grad öffentlicher Aufmerksamkeit, sondern nach »objektiven « wissenschaftlichen Kriterien eingestuft werden, die den ökologischen Risiken ebensoviel Bedeutung beimessen wie den Risiken für die menschliche Gesundheit.

2. Die wissenschaftlichen Kriterien für die obenstehende Klassifizierung der Umweltrisiken waren die geographischen Ausmaße des Umweltproblems, der benötigte Zeitraum zu ihrer Eindämmung bzw. ihre Irreversibilität. Daten und Methoden für die Bewertung von Umweltrisiken sollten weiterentwickelt werden.

3. Die festgestellten Risiken sollten sich in der langfristigen Planung der Behörde und auch in ihrem Budget ausdrücken. Weil die meisten Mittel allerdings direkt an die Implementation bestimmter Gesetze gebunden seien, müsse jeder erdenkliche Spielraum genutzt werden.

4. Das Science Advisory Board empfiehlt außerdem eine stärkere Ausschöpfung der instrumentellen Möglichkeiten in der Umweltpolitik, einschließlich Vorsorge und den Einsatz von ökonomischen Anreizmechanismen. Wirksam könne eine staatliche Umweltpolitik jedoch nur werden, wenn ihre Anliegen auch in die Agenda der übrigen Teile der Administration integriert würden.

5. Zu den dringend erforderlichen Verbesserungen zählt das Science Advisory Board Fortschritte in der Methodik zur Bewertung natürlicher Ressourcen: »Reliance on , willingness to pay a and similar techniques commonly used in economic analyses has distorted current understanding of the value of natural resources. « $7^{22}$

Diese Empfehlungen sind ausgesprochen ambitioniert, denn sie erfordern genau das, was der Behörde, wie weiter oben gezeigt, am meisten fehlt: Spielräume zur Verwirklichung einer "missionsorientierten « Umweltschutzpolitik. Zumindest auf die Art der Analyse haben die Empfehlungen der Science Advisory Board mittlerweile Einfluß gehabt. Trotzdem gibt es schon Beispiele für die Anwendung des Prinzips "relatives Risiko « zumindest in der Art der Analyse. Eine Arbeitsgruppe in der EPA hat sich mit der Frage befaßt, wie sich Umweltbelastungen auf die sozialen Schichtungssegmente und ethnischen Zugehörigkeiten verteilen. ${ }^{73}$ Es stellte sich eine weit überproportionale Belastung ethnischer Minderheiten und sozial Unterprivilegierter heraus durch Blei, verschiedene Luftschadstoffe, Altlasten, kontaminierte Nahrungsmittel (insbesondere in selbstgefangenem Fisch) und Pestizidanwendung für die in der Landwirtschaft Beschäftigten. Es bleibt abzuwarten, ob gegen die besondere Belastung bestimmter Bevölkerungsgruppen auch besonders intensive Umweltschutzbemühungen folgen werden.

\section{Schlußbemerkung}

Bedeutung und Einfluß von Regierungsinstitutionen hängen nicht allein von Größe und Budget $a b$, sondern auch von »politischen Realitäten «. ${ }^{74}$ Dieser Text interessierte sich für einige Aspekte dieser »Realitäten « im Bereich Umweltpolitik in den USA.

I. Nach mehr als zwanzig Jahren verfügt die EPA noch immer nicht über eine konsistente Organisationsstruktur. Das ist in erster Linie ein hausgemachtes Problem,

72 Environmental Protection Agency (Fn. 71), S. 8.

73 Environmental Protection Agency (Hrsg.) (1992): Environmental equity: Reducing risk for all communities. Volume 1: Workgroup report to the Administrator. Policy, Planning, and Evaluation. Washington D. C.

74 Rosenbaum (Fn. 27), S. 88. 
aber auch Ausdruck des nach wie vor herrschenden politischen Problemverständnisses und gesetzlicher Steuerungsversuche in der Umweltpolitik.

2. Das für das Regierungssystem der Vereinigten Staaten charakteristische System der »checks and balances« zwischen Exekutive und Legislative mit häufig gegenläufiger Interessenlage zwängt eine missionsorientierte Behörde wie die EPA in einen "Cycle of Distrust«, in dem umweltpolitische Entscheidungsspielräume durch die Aktoren des pluralist regime, von Weißem Haus, Kongreß und »special interests «, usurpiert werden. Eine seit Jahren diskutierte Aufwertung der EPA in den Rang eines Ministeriums könnte einen Beitrag zu mehr Unabhängigkeit leisten.

3. Die Erfahrungen mit dem emissions trading in den Vereinigten Staaten zeigen, daß die rechtlich-administrativen Voraussetzungen in der Diskussion um den Einsatz umweltökonomischer Instrumente gerne unterschätzt werden und ihre tatsächliche Attraktivität für Unternehmen nicht immer ausreichend hinterfragt wird. Rechtliche Definition und Vergabemodus der Verschmutzungsrechte, in erster Linie aber der große Informationsbedarf sind die Hauptschwierigkeiten bei der Implementation der bislang angewandten Modelle. Der Einsatz von ökonomischen Anreizmechanismen in der Umweltpolitik wird durch die Novellierung des Clean Air Act von 1990 forciert. Die EPA selbst versucht darüber hinaus das Prinzip ökonomischer Anreize auch auf andere Gebiete der Umweltpolitik zu uibertragen.

Die defensive Rolle in der Umweltpolitik zumindest teilweise abzulegen - das ist ein wesentlicher Beweggrund für das Nachdenken über »relative risk « als strategisches Leitbild bei der Umweltbehörde. Dort, wo die größten Minderungen von Umweltrisiken zu erwarten sind, da sollen die eigenen Ressourcen eingesetzt werden. Mit einem »objektiven «, wissenschaftlich hergeleiteten Risikobegriff soll die Befreiung aus der institutionellen Gefangenschaft und vom defensiven »Nur-Reagieren " gelingen. Ein solcher objektiver Risikomaßstab ist noch nicht entwickelten. Und in dem beschriebenen politisch-institutionellen Umfeld werden die umweltpolitischen Konsequenzen einer »objektivierten«Prioritätensetzung nicht leicht $\mathrm{zu}$ verwirklichen sein.

\section{Helmut Görling \\ Unternehmensdeals und Minderheitsaktionäre in der Ökonomischen Analyse des Rechts*}

"Mergers \& Acquisitions«, wie man das Geschäft mit den Unternehmensdeals nennt, ist ein Thema mit großer Bedeutung in der Wirtschaftspraxis'. Im Zukauf von Unternehmen wird in den Führungsetagen von Großkonzernen und auch mittelständischer Unternehmen in einer Zeit rascher technologischer Innovationen, wachsenden Wettbewerbsdrucks, gewaltiger Forschungs- und Entwicklungskosten und

\footnotetext{
* Zugleich eine Besprechung von Jurgen Reul: Die Pflicht zur Gleıchbehandlung der Aktionare bei privaten Kontrolltransaktionen: eine juristische und okonomische Analyse (Tübinger rechtswissenschaftliche Abhandlungen; Bd. 70); Tubingen: Mohr 1991, 344 Seiten, I68,- DM

I Im letzten Jahr wurden 2559 Firmenkaufe mit deutscher Beteiligung gezahlt; vgl. Frankfurter Rundschau vom 14. 4. 1993, S. 14. In Deutschland gingen 1635 Deals im Wert von 57 Mrd. DM uber die Buhne; vgl. Frankfurter Allgemene Zeitung vom 26. 4. 1993, S. B r 3 .
} 